\title{
Laser Power Beaming for Lunar Night and Permanently Shadowed Regions
}

Jonathan Grandidier

818-354-1566

Jet Propulsion Laboratory

jonathan.grandidier@jpl.nasa.gov

Co-Authors

Paul Jaffe, U.S. Naval Research Laboratory William T. Roberts, Jet Propulsion Laboratory

Malcolm W. Wright, Jet Propulsion Laboratory

Abigail A. Fraeman, Jet Propulsion Laboratory

Carol A. Raymond, Jet Propulsion Laboratory

Alex Austin, Jet Propulsion Laboratory

Philip Lubin, University of California, Santa Barbara

Eric T. Sunada, Jet Propulsion Laboratory

John-Paul Jones, Jet Propulsion Laboratory

Ansel Barchowsky, Jet Propulsion Laboratory

John D. Baker, Jet Propulsion Laboratory 
Laser Power Beaming for Lunar Night and Permanently Shadowed Regions

Jonathan Grandidier ${ }^{1}$, Paul Jaffe ${ }^{2}$, William T. Roberts ${ }^{1}$, Malcolm W. Wright ${ }^{1}$, Abigail A. Fraeman ${ }^{1}$, Carol A. Raymond ${ }^{1}$, Alex Austin ${ }^{1}$, Philip Lubin ${ }^{3}$, Eric T. Sunada ${ }^{1}$, John-Paul Jones ${ }^{1}$, Ansel Barchowsky ${ }^{1}$ and John D. Baker ${ }^{1}$

${ }^{1}$ Jet Propulsion Laboratory, California Institute of Technology, ${ }^{2}$ U.S. Naval Research Laboratory, ${ }^{3}$ University of California in Santa Barbara

Laser power beaming involves the wireless transfer of power [1, 2], and could provide a revolutionary new way to power robotic systems in permanently shadowed regions (PSR) or a base during the lunar night (Fig. 1). It has the potential to represent an alternative solution to provide power where sunlight is unavailable and could power systems such as Pop-Up Flat Folding Explorer Robot (PUFFER) or Cooperative Autonomous Distributed Robotic Explorers (CADRE) [3]. This paper will discuss power beaming applications on the Moon. We will then describe current power beaming development for the military and for deep space. Finally, we will detail some of the major subsystems needed for an end-to-end laser power beaming system.

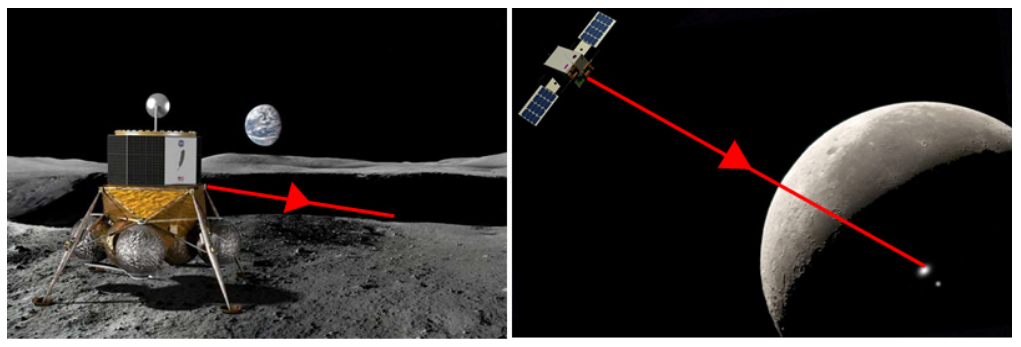

Figure 1. Power beaming to a PSR. Credit: Blue Origin (left) or to a lunar base during lunar night (right).

\section{Power beaming applications on the Moon}

\section{a. Lunar application}

Laser power beaming could provide power for robotic lunar missions to regions that have limited or no sunlight. One such location would be polar PSRs, which contains water ice, volatiles, and possibly organics. These areas may act as a witness plate to the history of volatile flux throughout the inner solar system, and also provide potential for in situ resource utilization. In situ measurements needed to address key questions related to volatiles trapped in PSRs include determining their elemental, mineralogical, and isotopic compositions, characterizing the lunar atmosphere in proximity to the cold traps, and mapping their spatial and temporal variability [4]. Several of these measurements, such as determining the chemical and isotopic compositions of volatiles within PSRs, could require in situ assets to survive in PSRs for extended period of time in order to access volatile deposits and make necessary measurements. This could be enabled by a lander on the rim of a crater that carries a laser power station which powers a rover. There are likely to be some visibility constraints due to the need to be at line of sight based on the location of the lander, but a relatively large percentage of the crater would still be accessible.

Laser power beaming may also be possible from lunar orbit to the surface, providing essential power for key assets during the long lunar night. Lunar geophysical networks are planned to collect long-term data sets to characterize the Moon's internal structure, seismic activity, global heat flow budget, bulk composition, and magnetic field. Measurements through the lunar night provide key constraints on the Moon's deep structure by 1) monitoring long-period induced 
signals from the lunar core driven by the Moon's interaction with the dynamic solar wind and Earth's magnetosphere; and 2) obtaining a complete record of moonquakes. These measurements would help provide insight into the thermal evolution of the lunar crust, mantle, and core, and help constrain the timeline of the ancient lunar dynamo. In addition, it would be possible to observe temporally-changing phenomena across the lunar day/night cycle critical for investigating the influence of the plasma and electromagnetic environment on the exosphere and volatile cycling.

\section{b. Robotic Lunar Surface Operations 2 (RLSO2)}

While power beaming undoubtedly has many applications for robotic science exploration, it also has the potential to enable future human exploration and industrial initiatives on the lunar surface. Discoveries in the last 20 years have shown that the poles of the Moon have abundant volatiles, including water ice, which could be harvested for use as propellant [5]. The highest concentrations of these volatiles exist in permanently shadowed craters, but transferring power into these regions of complete darkness is a significant challenge, as running large power transmission cable down the steep crater walls may be difficult or impossible. Laser power beaming presents a potentially enabling approach to accessing the volatiles in PSRs. Recent studies have considered different architecture implementation options for a large scale propellant-producing base at the South Pole of the Moon [6].

\section{Current power beaming development}

\section{a. An emerging game-changing technology for military applications}

Many military energy applications are hamstrung by dependence on fossil fuels, batteries with limited life and capacity, and operations in areas that are difficult, expensive, and risky for energy resupply via conventional means. Within the past few years, there has been a surge in advances in multiple technology areas that have direct and compelling relevance to these problems. Technology to support wireless transmission of energy over long distances has gained momentum, and recognition by many entities [7]. An emerging game-changing technology for military applications at its heart, the motivation for power beaming is its ability, in contexts unsuitable for wires or fuel, to transfer energy from a place where it is comparatively easy to generate or store to a place where it is needed. Power beaming may offer compelling crossover opportunities with other areas of military relevance such as directed energy (DE), electronic warfare, and communications. If successful, it may also present critical and disruptive opportunities to ensure forces have decisive overmatch in key areas that are undergirded by reliable energy as it enables new operational concepts.

\section{b. Long range deep space applications}

Large scale DE offers the possibility for radical transformation of a variety of areas including long range beamed power, rapid interplanetary travel and the future ability to achieve relativistic flight. DE relies on photonics, which like electronics is an exponentially expanding growth area driven by diverse economic interests that allows transformational advances in space exploration and capability. This capability is synergistic with conventional power sources (small 
fission, solar PV during the lunar day) and offers a road to a future currently not possible with conventional capabilities. We have currently achieved greater than $42 \%$ wall plug efficiency at UCSB for fiber laser systems, limited by pump diode conversion efficiency, which is currently $55 \%$. Using modular and scalable technology, such systems are applicable over a vast range from across a room to across the solar system in the long term. This also fits well with the desire to use the moon as a "gateway" to projecting power to much larger distances from Earth.

\section{Laser power beaming end- to-end system}

Laser power beaming technology and systems are at a low technology readiness level (TRL), and many technical challenges need to be resolved before it can be baselined for future missions. There is a need to develop efficient endto-end power systems from the

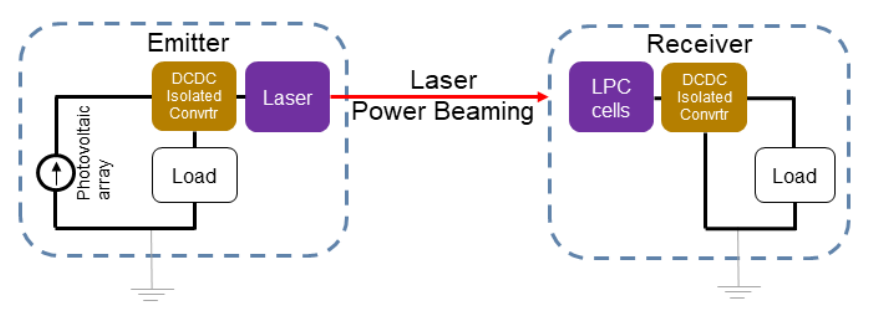

Figure 2. Block diagram of the end-to-end power beaming system. The emitter can be located on an orbiting spacecraft or at the rim of a PSR. The receiver is a lunar base or a robotic system in a PSR. transmitter to the receiver (Fig. 2), as well as new mission concepts and architectures that would take full advantage of these new capabilities. Here we describe some of the major subsystems.

\section{a. Laser source}

The laser source for power beaming applications can be tailored to the mission required. For near range requirements (up to a few hundreds of meters), high power semiconductor diode lasers can provide sufficient power in compact and power efficient packages. Commercial systems routinely are available across the wavelength range with 100's W available particularly around $1 \mu \mathrm{m}$. For longer range applications, where the optical beam properties are important to ensure power delivered on the target arrays, fiber lasers are most applicable due to their high brightness, with single mode output beams and extremely high powers leveraged from the industrial machining market. Multi-kW average powers at 1.06 um are possible in compact packages with 20-30\% efficiencies and weight about $20 \mathrm{~kg}$. An example shown in Fig. 3 is a commercial $2 \mathrm{~kW}$ high power fiber amplifier in a $24 \times 14 \times 2.25 \mathrm{in}^{3}$ with an electrical efficiency of $33 \%$ and diffraction limited output beam. Scaled down lasers at lower powers also exist, such as a $200 \mathrm{~W}$ laser in a $5 \times 13 \times 2$ inch $^{3}$ package with similar power efficiencies. Given the high powers, thermal management is critical as discussed in section III $\mathrm{g}$.

\section{b. Optical element}

The relationship between the beam radius at its narrowest point (the beam waist) and the distant beam is given by the equation $w(z)=$ $w_{0}\left[1+\left(\frac{z}{z_{r}}\right)^{2}\right]^{1 / 2}$ where $w(z)$ is the beam radius at distance $z, w_{0}$ is the beam waist radius, and $z_{r}$ is the beam's Rayleigh range $\left(\pi \omega_{0}^{2} / \lambda\right)$. This relationship determines the most efficient location to place the beam

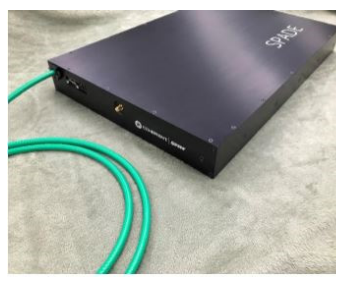

Figure 3. Fiber laser. waist and the resulting aperture diameters. 
Efficient designs for projecting power on the lunar surface (15 $\mathrm{km}$ range): The most costefficient design for a short-range power beaming system is heavily weighted toward reducing the beam radius at the transmitter at the expense of a larger receiver. Transmitters should be designed about 3 times the beam radius to limit diffraction losses, increase in cost with diameter cubed, and require precision pointing. None of these factors apply to a simple photovoltaic receiver. The design space between the transmit system and collection system is shown in Fig. 4a, in which we compare the diameter of the transmit aperture ( 3 times the beam radius) and the receive aperture ( 2 times the beam radius) for a power-beaming system at $1064 \mathrm{~nm}$. It shows apertures defined by placing the beam waist at (1) the receive aperture (blue line), (2) the transmit aperture (red line) and (3) an intermediate location between the two ends (yellow line). We have also included the point (black) at which the apertures have the same diameter (202 $\mathrm{mm})$, which occurs at the intersection of the yellow and red curves. The minimum cost system (for the same efficiency) will lie along the lower stretch of the red curve (smaller transmitter and larger collector).
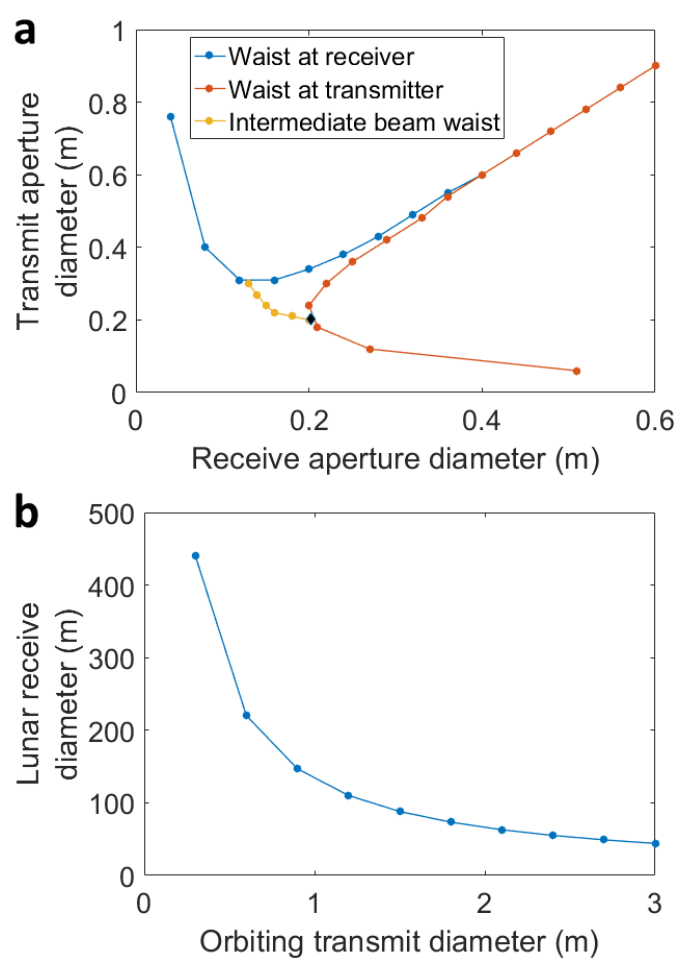

Figure 4. Design space between the transmit system and collection system for the a) surface to surface (black dot shows equal aperture case) and b) orbit to surface.

\section{Orbit to lunar surface power transfer:}

Here the distance over which the beam must be transmitted is much greater, so beam divergence will dictate that the beam waist be located at the transmit aperture for any reasonably sized aperture. A 1-meter transmit aperture on the orbiting system (already an expensive proposition) would emit a 2 rad diffraction-limited beam, but after propagation 65,000 km, the spot it generates on the moon is over 100 meters wide (Fig. 4b). Just about the only way of reducing this is to use a shorter wavelength laser, which, for the product of aperture diameters would decrease linearly with wavelength.

Compact, diffraction-limited transmit telescope designs such as the on-axis and off-axis Cassegrain telescopes shown in Fig. 5 are straightforward to design and fabricate. The more-conventional on-axis system is generally easier to manufacture, but suffers from the

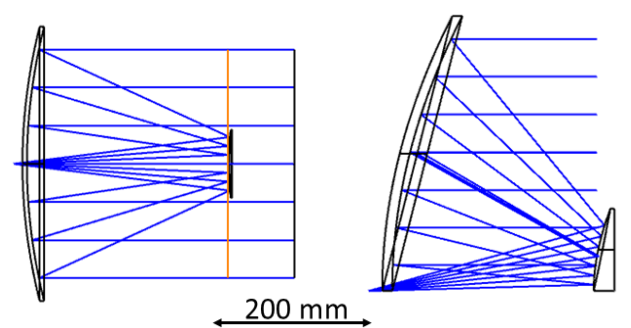

Figure 5. Compact, diffraction-limited transmit telescope designs such as the on-axis (left) and offaxis (right) Cassegrain telescopes technology. obscuration of the secondary mirror which blocks the brightest part of the transmit beam. If this design is to be used, the fiber output should be collimated soon after exit from the fiber, allowing the secondary mirror to be much smaller and 
recovering a large fraction of the obscuration loss. The-off-axis design avoids this loss, either in part as shown in Fig. 5, or in whole by displacing the two mirrors farther from the output axis of the beam. A $20 \mathrm{~cm}$ aperture $\mathrm{SiC}$ telescope would weight about $10 \mathrm{~kg}$.

Another critical element of the power beaming system is the need to accurately point the laser transmitter to ensure optimum power efficiency for the receiving element. Though no tracking is needed for ground based systems since both the transmitting source and receiver can be stationary during power beaming, accurate pointing knowledge and control is essential. A gimbaled control system for the optical transmit telescope that can deliver on the order of 1- 10 $\mu \mathrm{rad}$ pointing resolution is required. Such a pointing control system is readily available and has been demonstrated [8] on free space optical communications systems, but requires either a reference beacon from the receiver or knowledge of the receiver position through either ephemeris tracking in orbit or a ground radio tracking feature.

\section{c. Thermal management}

To generate a $1 \mathrm{~kW}$ optical laser beam, $3 \mathrm{kWe}$ powers a $33 \%$ efficient fiber laser and $2 \mathrm{~kW}$ of heat needs to be rejected. This occurs over an area of about $0.1 \mathrm{~m}^{2}$ that must be maintained no warmer than $40{ }^{\circ} \mathrm{C}$ to remain with specified laser limits. We assume an environmental

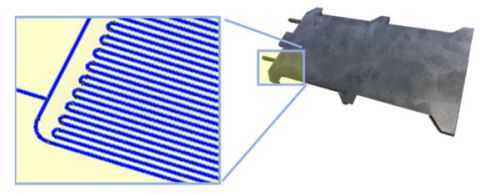

Figure 6. Multi-functional radiator with embedded oscillating heat pipe technology. sink temperature in the PSR $\leq 90 \mathrm{~K}$. To manage this $2 \mathrm{~W} / \mathrm{cm}^{2}$ flux such that temperature limits are maintained while also efficiently transporting the total $2 \mathrm{~kW}$ load over a radiator for ultimate heat rejection, integrated oscillating heat pipe (OHP) technology will be employed. The combination of forced convection and latent heat transfer associated with the OHP closed system is especially suitable for managing high heat fluxes [9]. Fig. 6 shows an example of an additively manufactured OHP that can be customized to accommodate the laser hardware [10]. OHPs are also extremely efficient heat spreaders over large areas. A radiator of approximate area $4.1 \mathrm{~m}^{2}$ is required for continuous laser operation (note: the radiator foot-print may be smaller if both sides can be utilized), and it will also include an OHP, which may be the same as that accommodating the laser, depending on configuration and manufacturability. $6 \mathrm{~kg} / \mathrm{m}^{2}$ is a recognized metric for thermal management for fluid space radiators.

\section{d. Laser power converter (LPC)}

The laser power converter is a semiconductor device that converts optical energy to electrical energy. It is optimized for high energy density and conversion efficiency under laser illumination. For a $1064 \mathrm{~nm}$ laser, InGaAs would be the optimal semiconductor with a bandgap of $1.1 \mathrm{eV}(1130 \mathrm{~nm})$. We at JPL have demonstrated a converted electrical power density of about $12 \mathrm{~kW} / \mathrm{m}^{2}$ for a InGaAs LPC [3]. This corresponds to about 30 times a regular solar array power output at $1 \mathrm{AU}$. Target optical to electrical conversion efficiency for the LPC is 65\%. For a $1 \mathrm{~kW}$ and $1 \mathrm{~m}^{2}$ LPC array, mass density would be similar to a solar array, around $2 \mathrm{~kg} / \mathrm{m}^{2}$.

\section{e. Battery}

Development of high specific energy, robust batteries for space applications has progressed dramatically over the past few decades, and JPL has been heavily involved in 
designing cells for both low temperature and high power robotic space applications [11]. Commercially available 18650 Li-ion cells like the LG Chem MJ1 cell can now achieve $>200 \mathrm{Wh} / \mathrm{kg}$ and $700 \mathrm{~W} / \mathrm{kg}$. The high energy density combined with the high power density and robust design led to their selection and qualification for the Europa Clipper mission. Higher power batteries are available as well, such as the Sony VTC4, which can operate at up to $30 \mathrm{~A}$ in an 18650 format, or $>2000 \mathrm{~W} / \mathrm{kg}$. A battery pack would require about 6.2 hours to charge fully assuming a $1 \mathrm{~kW}$ power source operating at $65 \%$ efficiency $(650 \mathrm{~W})$ and with a total energy of $4 \mathrm{kWh}$. At this low charging rate, high energy cells could be used. Taking a conservative estimate of $110 \mathrm{Wh} / \mathrm{kg}$ for the battery pack with high energy cells and de-ratings included, the mass of the energy storage system would be $\sim 36 \mathrm{~kg}$. A battery pack this size could easily provide $>250 \mathrm{~W}$ for roving operations 20 hours per day.

f. Power electronics

Power conversion electronics for high-powered, fiber-based, laser power transmission systems consist of two distinct converter types, which must be developed for spacecraft applications to ensure robust performance in harsh environments. First, to provide requisite current to the photodiodes in the diode or fiber laser, high currents at low voltages must be sourced. With diode forward voltage drops in the $2-10 \mathrm{~V}$ range, ampacity requirements for power conversion to the laser can reach $1.5 \mathrm{kA}$ for steady state power transmission on a $3 \mathrm{~kW}$ laser system. While such ampacities are readily achievable in commercial laser drivers, the challenges posed by both thermal dissipation and converter control for such systems in spacecraft applications are significantly more challenging [12]. Two general approaches exist, namely developing multilevel or multiphase converter systems $[13,14]$, both of which can be extended to spacecraft environments. An example multiphase system is shown in Fig. 7a, which is the flight hardware for the Lunar Flashlight diode instrument. Multiphase systems benefit from reduced switch stress, magnetics size, and filter size at the cost of increased control complexity. By paralleling phase-shifted power stages, modules can be designed into the multi-kW range [15], which can meet the needs of the proposed power beaming systems. Alternatively, multilevel converters such as the flying capacitor multilevel (FCML) converter, provide superior efficiency over the wide conversion ratios experienced in laser power systems, and are easily scalable to the multi-kW range through parallel stages. They have been demonstrated by JPL for space applications at efficiency up to $97 \%$, power density of $2 \mathrm{~W} / \mathrm{cm}^{3}$, and specific power of $1 \mathrm{~kW} / \mathrm{kg}$, providing a promising option for future laser power systems.

For processing power from the LPC to the load, similar technology can be leveraged to those under development for nextgeneration photovoltaic power generation for small spacecraft. With adaptive maximum power point tracking (MPPT) and GaNbased, digitally controlled buck-boost converters (Fig. 7b), power generated by the LPC can manage spacecraft loads and provide charge control to batteries on the payload system. Such converters have been demonstrated to TRL 3, processing $500 \mathrm{~W}$ at $96 \%$ efficiency in a single 3U PCB module [16].

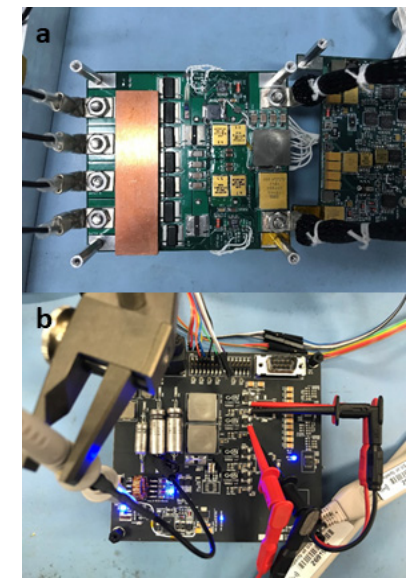

Figure 7. a) Laser power supply for Lunar Flashlight. b) MPPT buck-boost for PV Processing. 


\section{Conclusion}

Power beaming represents a game changing opportunity for space exploration, in particular in permanently shadowed regions or during the lunar night where sunlight is unavailable. In this paper, we described potential space mission scenarios that could benefit from power beaming. All the major subsystems of the power beaming system were considered. Measurements carried out at JPL show promising results for laser power transmission in the near infrared, where beam divergence can be limited. The research was carried out at the Jet Propulsion Laboratory, California Institute of Technology, under a contract with the National Aeronautics and Space Administration (80NM0018D0004), at the U.S. Naval Research Laboratory and at the University of California, Santa Barbara. (C) 2020. All rights reserved.

\section{References}

1. Sprangle, P., et al., High-power lasers for directed-energy applications. Applied Optics, 2015. 54(31): p. F201-F209.

2. Jin, K. and W. Zhou, Wireless Laser Power Transmission: A Review of Recent Progress. IEEE Transactions on Power Electronics, 2019. 34(4): p. 3842-3859.

3. Grandidier, J., et al. Power Beaming for Deep Space and Permanently Shadowed Regions. in The 2nd Optical Wireless and Fiber Power Transmission Conference (OWPT2020). 2020. Yokohama, Japan.

4. Council, N.R., The Scientific Context for Exploration of the Moon. 2007, Washington, DC: The National Academies Press. 120.

5. Colaprete, A., et al., Detection of Water in the LCROSS Ejecta Plume. Science, 2010. 330(6003): p. 463-468.

6. Austin, A., et al. Robotic Lunar Surface Operations 2. in International Astronautical Congress IAC2019. 2019. Washington DC, USA.

7. Jaffe, P., et al., Opportunities and Challenges for Space Solar for Remote Installations. 2019.

8. Boroson, D., et al., Overview and results of the Lunar Laser Communication Demonstration. SPIE LASE. Vol. 8971. 2014: SPIE.

9. Smoot, C.D. and H.B. Ma, Experimental Investigation of a Three-Layer Oscillating Heat Pipe. Journal of Heat Transfer, 2014. 136(5).

10. Daimaru, T., et al., Comparison between numerical simulation and on-orbit experiment of oscillating heat pipes. International Journal of Heat and Mass Transfer, 2017. 109: p. 791-806.

11. Bugga, R.V. and E.J. Brandon, Energy Storage for the Next Generation of Robotic Space Exploration. Electrochemical Society Interface, 2020. 29(1): p. 59-63.

12. Barchowsky, A., et al., A Class of GaN-Based, Radiation-Hardened Power Electronics for Jovian Environments. 2019. 3339-3345.

13. Magallanes, F.C., et al. Review of design solutions for high performance pulsed power converters. in 2012 15th International Power Electronics and Motion Control Conference (EPE/PEMC). 2012.

14. Kiani, M.H. and J.T. Stauth. Optimization and comparison of hybrid-resonant switched capacitor DC-DC converter topologies. in 2017 IEEE 18th Workshop on Control and Modeling for Power Electronics (COMPEL). 2017.

15. Reusch, D.C., High Frequency, High Power Density Integrated Point of Load and Bus Converters. 2012, Virginia Polytechnic Institute and State University, Blacksburgh, VA.

16. Barchowsky, A., et al. GaN-Based Ultra-Compact, Power Conversion System for the PUFFER Autonomous Mobility Platform. in AIAA SmallSat Conference. 2020. Logan, UT. 